Article

\title{
Synthesis and Biological Evaluation of Novel Acenaphthene Derivatives as Potential Antitumor Agents
}

\author{
Yong-Mei Xie $^{\dagger}$, Yi Deng ${ }^{\dagger}$, Xiao-Yun Dai, Jie Liu, Liang Ouyang, Yu-Quan Wei * and \\ Ying-Lan Zhao*
}

State Key Laboratory of Biotherapy, West China Hospital, West China Medical School, Sichuan University, Chengdu, 610041, China; E-Mails: xieym@scu.edu.cn (Y.M.X.); xiaofeng2525@126.com (Y.D.); homcndc@163.com (X.Y.D); 1jscsx2009@163.com (J. L.); klivis@163.com (L. O.)

$\dagger$ These authors contributed equally to this work.

* Authors to whom correspondence should be addressed; E-Mails: yuquawei@vip.sina.com (Y.-Q.W.); alancenxb@sina.com (Y.-L.Z.); Tel.: +86-28-85164063; Fax: +86-28-85164060.

Received: 15 February 2011; in revised form: 02 March 2011 / Accepted: 10 March 2011 / Published: 17 March 2011

\begin{abstract}
Twelve novel acenaphthene derivatives have been synthesized. The structures of all compounds were confirmed by ${ }^{1} \mathrm{H}-\mathrm{NMR}$, MS and elemental analysis. Their antitumor activities were evaluated in six human solid tumor cell lines, namely non-small cell lung cancer (H460), human colon adenocarcinoma (SW480), human breast cancer cell (MDA-MB-468 and SKRB-3), human melanoma cell (A375) and human pancreatic cancer (BxPC-3) . Among them, compound 3c shows the best antitumor activity against SKRB-3 cell line, as high as the positive control adriamycin.
\end{abstract}

Keywords: synthesis; acenaphthene derivatives; antitumour activity

\section{Introduction}

Acenaphthene derivatives have gained great importance due to their diverse biological properties including antitumor [1,2], antifungal [3], antimicrobial [4], anti-inflammatory [5,6] and insecticidal [7] activities. In order to obtain novel acenaphthene derivatives with a wide spectrum of pharmaceutical applications, we report herein the synthesis of a series of acenaphthene derivatives containing thiazole backbone and the results of their preliminary in vitro antitumor evaluation. 


\section{Results and Discussion}

\subsection{Chemistry}

The synthetic route for the target compounds is shown in Scheme 1. The prepared acenaphthene derivatives 3a-h and $\mathbf{4 a - d}$ are listed in Table 1.

Scheme 1. Synthetic route for 3a-h and 4a-d.

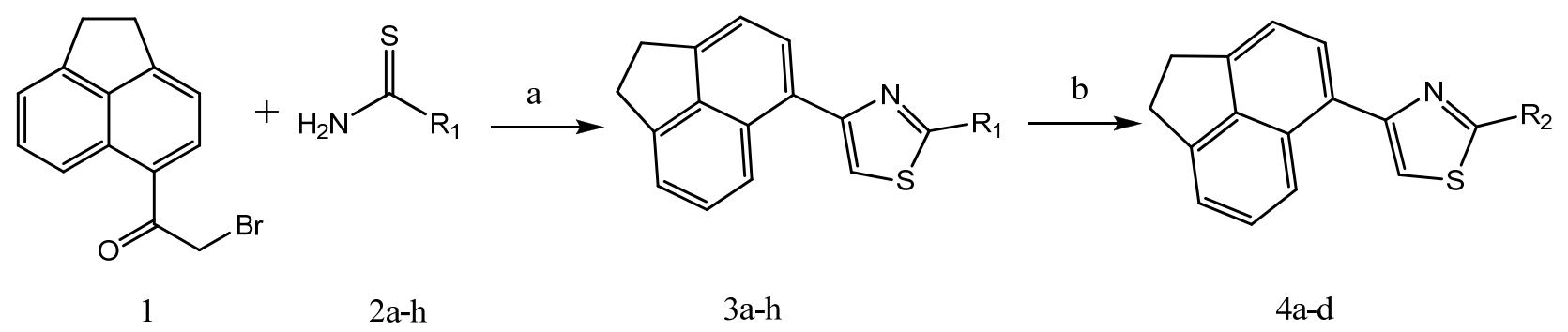

Reagents and conditions: a) ethanol, reflux, $2-5 \mathrm{~h}, \mathrm{NaHCO}_{3}$; b) acyl chloride, ethyldiisopropylamine, pyridine, reflux, 6-8 $\mathrm{h}$.

Commercially available acenaphthene was first reacted with bromoacetyl bromide in the presence of aluminum chloride to give crude 5-bromoacetylacenaphthene (1) (containing a little 3-bromoacetyl acenaphthene). The raw product was purified using a silica column with ethyl acetate/petroleum ether $(1: 15 \mathrm{v} / \mathrm{v})$ as eluent. 3-Bromoacetylacenaphthene was eluted first and 5-bromoacetylacenaphthene was elueted next. The compounds 3a-h were prepared by reacting $\mathbf{1}$ and 2-thiourea (2a) or aryl thioureas 2b-h under reflux using ethanol as solvent. The $\mathrm{HBr}$ produced was neutralized with $\mathrm{NaHCO}_{3}$ solution and the crude product was recrystallized from appropriate solvent to yield 3a-h (purity >97\%, HPLC). Compound 3a was further reacted with an appropriate acyl chloride in the presence of ethyldiisopropylamine using pyridine as solvent. Compounds $4 \mathbf{a}-\mathbf{b}$ (purity $>97 \%$, HPLC) were obtained by recrystallization of the crude products from appropriate solvents without purification by column chromatography.

\subsection{Antiproliferative Activities}

In the present study, the antiproliferative activity of the synthesized compounds was tested in vitro on six human tumor cell lines, including non-small cell lung cancer (H460), human colon adenocarcinoma (SW480), human breast cancer cell (MDA-MB-468 and SKRB-3), human melanoma cell (A375) and human pancreatic cancer (BxPC-3) by the MTT assay with adriamycin (ADM) as a positive control. The inhibition rates under drug concentration of $20 \mu \mathrm{M}$ are summarized in Table 1 . Among the acenaphthene derivatives, 3c shows better inhibition against breast cancer cells line of MDA-MB-468 and SKRB-3 (inhibition rate are 55.5 $\pm 3.8 \%$ and $66.1 \pm 2.2 \%$ respectively), while the corresponding results for ADM are $63.4 \pm 0.4 \%$ and $68.1 \pm 1.3 \%$. These results suggest that $3 c$ has potent antitumor activity. 
Table 1. The substituents and antitumor activities of 3a-h and 4a-d.*

\begin{tabular}{|c|c|c|c|c|c|c|c|c|}
\hline \multirow[b]{2}{*}{ Compd. } & \multirow[b]{2}{*}{$\mathbf{R}_{\mathbf{1}}$} & \multirow[b]{2}{*}{$\mathbf{R}_{2}$} & \multicolumn{6}{|c|}{ Inhibition rate (\%) } \\
\hline & & & H460 & SW480 & $\begin{array}{c}\text { MDA- } \\
\text { MB-468 }\end{array}$ & $\begin{array}{c}\text { SKRB- } \\
3 \\
\end{array}$ & A375 & BxPC-3 \\
\hline За & $-\mathrm{NH}_{2}$ & - & $25.2 \pm 2.8$ & $1.7 \pm 2.3$ & $0.8 \pm 3.9$ & $1.3 \pm 8.8$ & $42.3 \pm 2.2$ & $4.0 \pm 3.4$ \\
\hline $3 \mathbf{b}$ & & & $19.3 \pm 6.6$ & $20.2 \pm 5.6$ & $27.3 \pm 6.8$ & $35.2 \pm 3.2$ & $25.7 \pm 3.2$ & $18.6 \pm 2.8$ \\
\hline $3 c$ & & 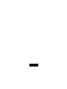 & $24.3 \pm 9.1$ & $22.6 \pm 3.0$ & $55.5 \pm 3.8$ & $66.1 \pm 2.2$ & $31.7 \pm 5.0$ & $17.1 \pm 3.7$ \\
\hline 3d & & & $12.2 \pm 4.0$ & $11.1 \pm 2.5$ & $31.6 \pm 8.1$ & $42.4 \pm 2.9$ & $18.2 \pm 9.2$ & $6.1 \pm 5.5$ \\
\hline $3 \mathbf{e}$ & & & $20.6 \pm 5.2$ & $0.4 \pm 2.6$ & $15.2 \pm 7.3$ & $20.7 \pm 4.4$ & $0.5 \pm 1.5$ & $2.6 \pm 6.6$ \\
\hline $3 f$ & & & $33.8 \pm 1.6$ & $30.7 \pm 0.5$ & $25.9 \pm 1.5$ & $47.5 \pm 2.8$ & $10.5 \pm 1.0$ & $20.6 \pm 2.4$ \\
\hline $3 g$ & & & $26.2 \pm 2.1$ & $1.9 \pm 2.6$ & $5.2 \pm 3.7$ & $34.7 \pm 6.6$ & $4.9 \pm 3.1$ & $1.1 \pm 7.8$ \\
\hline $3 \mathbf{h}$ & & & $43.7 \pm 1.3$ & $41.1 \pm 2.7$ & $30.6 \pm 0.6$ & $41.6 \pm 2.4$ & $9.1 \pm 1.6$ & $32.1 \pm 1.0$ \\
\hline $4 a$ & & & $12.1 \pm 0.5$ & $14.6 \pm 1.4$ & $20.5 \pm 1.6$ & $31.0 \pm 4.8$ & $22.6 \pm 2.5$ & $10.2 \pm 1.7$ \\
\hline $4 b$ & & & $17.6 \pm 0.8$ & $33.8 \pm 4.0$ & $31.3 \pm 1.3$ & $41.7 \pm 5.2$ & $35.0 \pm 6.6$ & $20.2 \pm 1.4$ \\
\hline 4c & & & $19.3 \pm 3.2$ & $16.3 \pm 0.4$ & $13.2 \pm 5.0$ & $26.3 \pm 3.0$ & $16.6 \pm 7.1$ & $11.4 \pm 3.6$ \\
\hline 4d & & & $35.1 \pm 3.3$ & $22.7 \pm 1.0$ & $25.3 \pm 1.9$ & $38.0 \pm 2.8$ & $39.7 \pm 1.9$ & $25.0 \pm 3.5$ \\
\hline ADM & & & $63.3 \pm 0.9$ & $46.1 \pm 0.4$ & $63.4 \pm 0.4$ & $68.1 \pm 1.3$ & $70.4 \pm 2.0$ & $39.4 \pm 0.7$ \\
\hline
\end{tabular}

* Results are given in concentrations of $20 \mu \mathrm{M}$ after a continuous exposure of $48 \mathrm{~h}$ and show means \pm SEM of three-independent experiments.

\section{Experimental}

\subsection{General}

The human cancer cell lines were purchased from the American Type Culture Collection (ATCC, Rockville, MD, USA). Dulbecco's modified Eagle medium (DMEM) and RPMI 1640 were purchased from Gibco (Grand Island, New York, USA). Fetal bovine serum (FBS) was purchased from Hyclone (Logan, Utah, USA). All chemicals were commercially available and used without further purification unless otherwise stated. Column chromatography was carried out on silica gel (200-300 mesh, Qingdao Marine Chemical Ltd., Qingdao, China). Thin layer chromatography (TLC) was performed on TLC silica gel 60 F254 plates. The purity of compound screened in biological assays was determined to be $\geq 97 \%$ by HPLC analysis with a photodiode array detector (Waters, Milford, MA, 
USA). An atlantis $\mathrm{C}_{18}(150 \mathrm{~mm} \times 4.6 \mathrm{~mm}$, i.d. $5 \mu \mathrm{m})$ (Waters, Milford, Mass, USA) was used with a gradient elution of methanol and HPLC-grade water as mobile phase at a flow rate of $1 \mathrm{~mL} / \mathrm{min} .{ }^{1} \mathrm{H}$ NMR were recorded at $400 \mathrm{MHz}$ on a Varian spectrometer (Varian, Palo Alto, CA, USA) model Gemini 400 and chemical shifts $(\delta)$ are reported in parts per million relative to tetramethylsilane (TMS) used as an internal standard, where $(\delta)$ TMS $=0.00 \mathrm{ppm}$. Mass Spectra (MS) were measured by Q-TOF Priemier mass spectrometer utilizing electrospray ionization (ESI) (Micromass, Manchester, UK). Elemental analyses were carried out on a Carlo Erba-1106 analyzer. Melting points were determined on a SGW X-4 microscopic melting point (Shanghai Precision \& Scientific Instrument Co., Ltd, China).

\subsection{Preparation of 5-Bromoacetylacenaphthene (1)}

5-Bromoacetylacenaphthene was synthesized according to a literature method [8] with some modifications. Briefly, aluminum chloride $(22.8 \mathrm{~g}, 171 \mathrm{mmol})$ was added slowly while stirring to a solution of acenaphthene $(18.6 \mathrm{~g}, 211 \mathrm{mmol})$ and bromoacetyl bromide $(12.6 \mathrm{~mL}, 145 \mathrm{mmol})$ in $\mathrm{CH}_{2} \mathrm{Cl}_{2}(120 \mathrm{~mL})$ at $-20{ }^{\circ} \mathrm{C}$. The mixture was allowed to warm to room temperature and stirred for $30 \mathrm{~min}$, neutralized with $5 \% \mathrm{NaHCO}_{3}$ solution, extracted with chloroform $(3 \times 50 \mathrm{~mL})$, washed with water $(2 \times 50 \mathrm{~mL})$ and dried over $\mathrm{Na}_{2} \mathrm{SO}_{4}$. The solvent was evaporated and residue was purified with column chromatography (ethyl acetate: petroleum ether $=1: 15$ ) to give compound $\mathbf{1}$ as an off-white solid. Yield: $13.8 \mathrm{~g}, 41.5 \%$. ${ }^{1} \mathrm{H}-\mathrm{NMR}\left(\mathrm{CDCl}_{3}\right) \delta: 3.43(\mathrm{~s}, 4 \mathrm{H}), 4.58(\mathrm{~s}, 2 \mathrm{H}), 7.33(\mathrm{~d}, 1 \mathrm{H}, J=7.6 \mathrm{~Hz})$, $7.40(\mathrm{~d}, 1 \mathrm{H}, J=6.8 \mathrm{~Hz}), 7.64\left(\mathrm{dd}, 1 \mathrm{H}, J_{1}=7.2 \mathrm{~Hz}, J_{2}=7.2 \mathrm{~Hz}\right), 8.09$ (d, 1H, J=7.6 Hz), 8.69 (d, 1H, $J=8.4 \mathrm{~Hz})$.

\subsection{General Procedure for Preparing Compounds 3a-h}

A mixture of compound 1 ( $0.50 \mathrm{mmol}), \mathbf{2 a}-\mathbf{h}(0.55 \mathrm{mmol}$; $\mathbf{2 b}-\mathbf{h}$ were prepared according to a literature method [9]) was refluxed in ethanol $(20 \mathrm{~mL})$. After completion of the reaction, the reaction mixture was evaporated. $5 \% \mathrm{NaHCO}_{3}$ solution $(5 \mathrm{~mL})$ was added to the residue and the mixture was stirred for $20 \mathrm{~min}$. The mixture was filtered and the obtained solid was recrystallized from solvent to give 3a-h.

4-(1,2-Dihydroacenaphthylen-5-yl)-1,3-thiazol-2-amine (3a). Off-white solid (from ethanol); Yield: 55 mg, 43.8\%; HPLC: 98.2\%; m.p. 155.8-157.3 ${ }^{\circ} \mathrm{C} ;{ }^{1} \mathrm{H}-\mathrm{NMR}$ (DMSO) $\delta: 3.46$ (m, 4H), 7.04 (s, 1H), $7.42\left(\mathrm{dd}, 2 \mathrm{H}, J_{1}=4.0 \mathrm{~Hz}, J_{2}=3.6 \mathrm{~Hz}\right), 7.56-7.81(\mathrm{~m}, 3 \mathrm{H}), 8.92(\mathrm{~s}, 2 \mathrm{H})$; LC-MS: $253.4(\mathrm{M}+\mathrm{H})^{+}$; Anal. Calcd. for $\mathrm{C}_{15} \mathrm{H}_{12} \mathrm{~N}_{2} \mathrm{~S}$ (252.07): C, 71.40; H, 4.79; N, 11.10\%. Found: C, 71.38; H, 4.81; N, 11.13\%.

N-Phenyl-4-(1,2-dihydroacenaphthylen-5-yl)-1,3-thiazol-2-amine (3b). White solid (from ethanol/ water = 1:1); Yield: $97 \mathrm{mg}, 59.1 \%$; HPLC: 98.8\%; m.p. 129.5-130.9 ${ }^{\circ} \mathrm{C} ;{ }^{1} \mathrm{H}-\mathrm{NMR}\left(\mathrm{CDCl}_{3}\right) \delta: 3.43(\mathrm{~d}$, $4 \mathrm{H}, J=2.0 \mathrm{~Hz}), 6.80(\mathrm{~s}, 1 \mathrm{H}), 7.06-7.09(\mathrm{~m}, 1 \mathrm{H}), 7.31-7.38(\mathrm{~m}, 5 \mathrm{H}), 7.49(\mathrm{~m}, 1 \mathrm{H}), 7.77(\mathrm{~d}, 2 \mathrm{H}, J=7.2$ $\mathrm{Hz}), 8.18(\mathrm{~d}, 1 \mathrm{H}, J=8.4 \mathrm{~Hz})$; LC-MS: $329.6(\mathrm{M}+\mathrm{H})^{+}$; Anal. Calcd. for $\mathrm{C}_{21} \mathrm{H}_{16} \mathrm{~N}_{2} \mathrm{~S}(328.43)$ : C, 76.80; H, 4.91; N, 8.53\%. Found: C, 76.83; H, 4.90; N, 8.52\%. 
$N$-(4-Ethoxyphenyl)-4-(1,2-dihydroacenaphthylen-5-yl)-1,3-thiazol-2-amine (3c). Gray solid (from ethanol); Yield:120 mg, 64.4\%; HPLC: 99.3\%; m.p. 194.3-197.0 ${ }^{\circ} \mathrm{C} ;{ }^{1} \mathrm{H}-\mathrm{NMR}\left(\mathrm{CDCl}_{3}\right) \delta: 1.43$ (t, $3 \mathrm{H}$, $J=6.8 \mathrm{~Hz}), 3.42\left(\mathrm{dd}, 4 \mathrm{H}, J_{1}=3.2 \mathrm{~Hz}, J_{2}=3.2 \mathrm{~Hz}\right), 4.03\left(\mathrm{dd}, 2 \mathrm{H}, J_{1}=6.8 \mathrm{~Hz}, J_{2}=7.2 \mathrm{~Hz}\right), 6.70(\mathrm{~s}$, $1 \mathrm{H}), 6.86(\mathrm{~d}, 2 \mathrm{H}, J=8.8 \mathrm{~Hz}), 7.24-7.31(\mathrm{~m}, 4 \mathrm{H}), 7.48(\mathrm{t}, 1 \mathrm{H}), 7.57(\mathrm{sbr}, 1 \mathrm{H}), 7.74(\mathrm{~d}, 1 \mathrm{H}, J=7.2 \mathrm{~Hz})$, $8.17(\mathrm{~d}, 1 \mathrm{H}, J=8.4 \mathrm{~Hz})$; LC-MS: $373.4(\mathrm{M}+\mathrm{H})^{+}$; Anal. Calcd. for $\mathrm{C}_{23} \mathrm{H}_{20} \mathrm{~N}_{2} \mathrm{OS}$ (372.48): C, 74.16; $\mathrm{H}$, 5.41; N, 7.52\%. Found: C, 74.15; H, 5.44; N, 7.51\%.

$N$-(4-Methoxyphenyl)-4-(1,2-dihydroacenaphthylen-5-yl)-1,3-thiazol-2-amine (3d). Gray solid (from ethanol); Yield: $94 \mathrm{mg}, 52.4 \%$; m.p. $185.4-188.6{ }^{\circ} \mathrm{C}$; HPLC: $99.6 \%$; ${ }^{1} \mathrm{H}-\mathrm{NMR}$ (DMSO) $\delta: 3.41(\mathrm{~s}, 4 \mathrm{H}$ ), $3.73(\mathrm{~s}, 3 \mathrm{H}), 6.94(\mathrm{~d}, 2 \mathrm{H}, J=8.8 \mathrm{~Hz}), 7.09(\mathrm{~s}, 1 \mathrm{H}), 7.36-7.62(\mathrm{~m}, 5 \mathrm{H}), 7.79(\mathrm{~d}, 1 \mathrm{H}, J=7.2, \mathrm{~Hz}), 8.22$ $(\mathrm{d}, 1 \mathrm{H}, J=7.2 \mathrm{~Hz}), 10.21(\mathrm{~s}, 1 \mathrm{H})$; LC-MS: $359.5(\mathrm{M}+\mathrm{H})^{+}$; Anal. Calcd. for $\mathrm{C}_{22} \mathrm{H}_{18} \mathrm{~N}_{2} \mathrm{OS}$ (358.46): C, 73.71; H, 5.06; N, 7.82\%. Found: C, 73.75; H, 5.07; N, 7.80\%.

$N$-(3,5-Dichlorophenyl)-4-(1,2-dihydroacenaphthylen-5-yl)-1,3-thiazol-2-amine (3e). White solid (from ethanol); Yield: $109 \mathrm{mg}, 54.9 \%$; HPLC: 98.6\%; m.p. 222.6-225.1 ${ }^{\circ} \mathrm{C} ;{ }^{1} \mathrm{H}-\mathrm{NMR}$ (DMSO) $\delta: 3.40$ $(\mathrm{s}, 4 \mathrm{H}), 7.13(\mathrm{~s}, 1 \mathrm{H}), 7.32(\mathrm{~s}, 1 \mathrm{H}), 7.37-17.54(\mathrm{~m}, 3 \mathrm{H}), 7.81(\mathrm{~d}, 1 \mathrm{H}, J=7.2 \mathrm{~Hz}), 7.85(\mathrm{~d}, 2 \mathrm{H}$, $J=1.6 \mathrm{~Hz}), 8.31(\mathrm{~d}, 1 \mathrm{H}, J=8.4 \mathrm{~Hz}), 10.78(\mathrm{~s}, 1 \mathrm{H})$; LC-MS: $397.3(\mathrm{M}+\mathrm{H})^{+}$; Anal. Calcd. for $\mathrm{C}_{21} \mathrm{H}_{14} \mathrm{~N}_{2} \mathrm{~S}$ (397.32): C, 63.48; H, 3.55; N, 7.05\%. Found: C, 63.45; H, 3.57; N, 7.06\%.

$\mathrm{N}$-(4-Chlorophenyl)-4-(1,2-dihydroacenaphthylen-5-yl)-1,3-thiazol-2-amine (3f). White solid (from ethanol); Yield: $105 \mathrm{mg}, 57.9 \%$; HPLC: 99.5\%; m.p. 211.4-214.5 ${ }^{\circ} \mathrm{C} ;{ }^{1} \mathrm{H}-\mathrm{NMR}\left(\mathrm{CDCl}_{3}\right)$ $\delta: 3.41$ (s, $4 \mathrm{H}), 6.78(\mathrm{~s}, 1 \mathrm{H}), 7.15-7.30(\mathrm{~m}, 5 \mathrm{H}), 7.47\left(\mathrm{dd}, 1 \mathrm{H}, J_{1}=7.2 \mathrm{~Hz}, J_{2}=7.2 \mathrm{~Hz}\right), 7.72(\mathrm{~d}, 2 \mathrm{H}, J=7.2 \mathrm{~Hz})$, $8.08(\mathrm{sbr}, 1 \mathrm{H}), 8.13(\mathrm{~d}, 1 \mathrm{H}, \quad J=8.4 \mathrm{~Hz})$; LC-MS: $363.5(\mathrm{M}+\mathrm{H})^{+}$; Anal. Calcd. for $\mathrm{C}_{21} \mathrm{H}_{15} \mathrm{ClN}_{2} \mathrm{~S}$ (362.88): C, 69.51; H, 4.17; N, 7.72\%. Found: C, 69.50; H, 4.19; N, 7.71\%.

4-(4-(1,2-Dihydroacenaphthylen-5-yl)-1,3-thiazol-2-ylamino) benzoic acid (3g). White solid (from ethanol); Yield: $137 \mathrm{mg}, 73.6 \%$; HPLC: 99.0\%; m.p.323.1-325.6 ${ }^{\circ} \mathrm{C}$; ${ }^{1} \mathrm{H}-\mathrm{NMR}$ (DMSO) $\delta: 3.40$ (s, 4H), $7.28(\mathrm{~s}, 1 \mathrm{H}), 7.38(\mathrm{~m}, 2 \mathrm{H}), 7.53\left(\mathrm{dd}, 1 \mathrm{H}, J_{1}=7.2 \mathrm{~Hz}, J_{2}=7.2 \mathrm{~Hz}\right), 7.80-7.91(\mathrm{~m}, 5 \mathrm{H}), 8.24(\mathrm{~d}, 1 \mathrm{H}$, $J=8.4 \mathrm{~Hz}), 10.74(\mathrm{~s}, 1 \mathrm{H}), 12.52(\mathrm{~s}, 1 \mathrm{H})$; LC-MS: $373.4(\mathrm{M}+\mathrm{H})^{+}$; Anal. Calcd. for $\mathrm{C}_{22} \mathrm{H}_{16} \mathrm{~N}_{2} \mathrm{O}_{2} \mathrm{~S}$ (372.44): C, 70.95; H, 4.33; N, 7.52\%. Found: C, 70.91; H, 4.35; N, 7.54\%.

Methyl 2-(4-(1,2-dihydroacenaphthylen-5-yl)thiazol-2-ylamino)benzoate (3h). White solid (from ethanol); Yield: $122 \mathrm{mg}, 63.1 \%$; HPLC: 99.1\%; m.p. 258.4-260.7 ${ }^{\circ} \mathrm{C}$; ${ }^{1} \mathrm{H}-\mathrm{NMR}$ (DMSO) $\delta: 3.43$ (s, $4 \mathrm{H}), 4.93(\mathrm{~s}, 3 \mathrm{H}), 7.15(\mathrm{~d}, 2 \mathrm{H}, J=8.0 \mathrm{~Hz}), 7.35-7.66(\mathrm{~m}, 5 \mathrm{H}), 8.00(\mathrm{~d}, 1 \mathrm{H}, J=8.0 \mathrm{~Hz}), 8.35(\mathrm{~d}, 1 \mathrm{H}$, $J=8.4 \mathrm{~Hz}), 8.46(\mathrm{~d}, 1 \mathrm{H}, J=7.2 \mathrm{~Hz}), 12.72(\mathrm{~s}, 1 \mathrm{H})$; LC-MS: $387.6(\mathrm{M}+\mathrm{H})^{+}$; Anal. Calcd. for $\mathrm{C}_{23} \mathrm{H}_{18} \mathrm{~N}_{2} \mathrm{O}_{2} \mathrm{~S}$ (386.47): C, 71.48; H, 4.69; N, 7.25\%. Found: C, 71.45; H, 4.70; N, 7.28\%.

\subsection{General Procedure for Preparing Compounds 4a-d}

A mixture of 3a $(0.50 \mathrm{mmol})$, acyl chloride $(0.55 \mathrm{mmol})$ and ethyldiisopropylamine $(0.3 \mathrm{mmol})$ was refluxed in pyridine $(10 \mathrm{~mL})$. After completion of the reaction, the reaction mixture was evaporated. Water $(20 \mathrm{~mL})$ and ethyl acetate $(50 \mathrm{~mL})$ were added. The organic layer was washed with $5 \% \mathrm{Na}_{2} \mathrm{CO}_{3}$ 
solution $(20 \mathrm{~mL})$, water and saturated sodium chloride solution, then dried over $\mathrm{Na}_{2} \mathrm{SO}_{4}$. The filtrate was evaporated and the obtained solid was recrystallized from solvent to give 4a-d.

$N$-(4-(1,2-dihydroacenaphthylen-5-yl)-1,3-thiazol-2-yl) acetamide (4a). White solid (from ethanol/ ethyl acetate $=2: 1)$; Yield: $86 \mathrm{mg}, 58.4 \%$; HPLC: $98.1 \%$; m.p. 192.5-193.8 ${ }^{\circ} \mathrm{C} ;{ }^{1} \mathrm{H}-\mathrm{NMR}\left(\mathrm{CDCl}_{3}\right) \delta$ : $1.73(\mathrm{~s}, 3 \mathrm{H}), 3.44(\mathrm{~s}, 4 \mathrm{H}), 7.13(\mathrm{~s}, 1 \mathrm{H}), 7.33\left(\mathrm{dd}, 2 \mathrm{H}, J_{1}=4.0 \mathrm{~Hz}, J_{2}=4.0 \mathrm{~Hz}\right), 7.47\left(\mathrm{q}, 1 \mathrm{H}, J_{1}=6.8 \mathrm{~Hz}\right.$, $\left.J_{2}=6.8 \mathrm{~Hz}\right), 7.72(\mathrm{~d}, 1 \mathrm{H}, J=7.2 \mathrm{~Hz}), 8.09(\mathrm{~d}, 1 \mathrm{H}, J=8.4 \mathrm{~Hz}), 10.70(\mathrm{~s}, 1 \mathrm{H})$; LC-MS: $295.4(\mathrm{M}+\mathrm{H})^{+}$; Anal. Calcd. for $\mathrm{C}_{17} \mathrm{H}_{14} \mathrm{~N}_{2} \mathrm{OS}$ (294 .37): C, 69.36; H, 4.79; N, 9.52\%. Found: C, 69.40; H, 4.78; N, $9.50 \%$.

$\mathrm{N}$-(4-(1,2-dihydroacenaphthylen-5-yl)-1,3-thiazol-2-yl)benzamide (4b). Off-white solid (from ethanol/ water = 4:1); Yield: $79 \mathrm{mg}, 44.3 \%$; HPLC: 98.6\%; m.p. 173.2-176.1 ${ }^{\circ} \mathrm{C} ;{ }^{1} \mathrm{H}-\mathrm{NMR}\left(\mathrm{CDCl}_{3}\right) \delta: 3.37$ (dd, $\left.4 \mathrm{H}, J_{1}=7.6 \mathrm{~Hz}, J_{2}=8.0 \mathrm{~Hz}\right), 7.19-7.43(\mathrm{~m}, 5 \mathrm{H}), 7.61(\mathrm{~d}, 1 \mathrm{H}, J=7.2 \mathrm{~Hz}), 7.72(\mathrm{~d}, 2 \mathrm{H}, J=7.6 \mathrm{~Hz})$, $8.01(\mathrm{~d}, 1 \mathrm{H}, J=8.4 \mathrm{~Hz}), 11.06(\mathrm{~s}, 1 \mathrm{H})$; LC-MS: $357.4(\mathrm{M}+\mathrm{H})^{+}$; Anal. Calcd. for $\mathrm{C}_{22} \mathrm{H}_{16} \mathrm{~N}_{2} \mathrm{OS}$ (356.44): C, 74.13; H, 4.52; N, 7.86\%. Found: C, 74.15; H, 4.50; N, 7.88\%.

$\mathrm{N}$-(4-(1,2-dihydroacenaphthylen-5-yl)-1,3-thiazol-2-yl)-4-methoxybenzamide (4c). White solid (from ethanol/ ethyl acetate $=2: 1$ ); Yield: $130 \mathrm{mg}, 67.3 \%$; HPLC: 99.5\%; m.p. 210.6-213.8 ${ }^{\circ} \mathrm{C}$; ${ }^{1} \mathrm{H}-\mathrm{NMR}$ $\left(\mathrm{CDCl}_{3}\right) \delta: 3.35\left(\mathrm{dd}, 4 \mathrm{H}, J_{1}=7.6 \mathrm{~Hz}, J_{2}=7.2 \mathrm{~Hz}\right), 3.73(\mathrm{~s}, 3 \mathrm{H}), 6.62(\mathrm{~d}, 2 \mathrm{H}, J=8.8 \mathrm{~Hz}), 7.17-7.28(\mathrm{~m}$, 3H), $7.40(\mathrm{~m}, 1 \mathrm{H}), 7.61(\mathrm{t}, 3 \mathrm{H}, J=7.2 \mathrm{~Hz}), 7.99$ (d, 1H, $J=8.4 \mathrm{~Hz}), 11.37$ (s, 1H); LC-MS: 387.5 $(\mathrm{M}+\mathrm{H})^{+}$; Anal. Calcd. for $\mathrm{C}_{23} \mathrm{H}_{18} \mathrm{~N}_{2} \mathrm{O}_{2} \mathrm{~S}$ (386.47): C, 71.48; H, 4.69; N, 7.25\%. Found: C, 71.47; H, $4.70 ; \mathrm{N}, 7.23 \%$.

$\mathrm{N}$-(4-(1,2-dihydroacenaphthylen-5-yl)-1,3-thiazol-2-yl)furan-2-carboxamide (4d). White solid (from ethanol/ ethyl acetate =2:1); Yield: $93 \mathrm{mg}, 53.7 \%$; HPLC: 98.7\%; m.p. 184.9-187.4 ${ }^{\circ} \mathrm{C}$; ${ }^{1} \mathrm{H}-\mathrm{NMR}$ $\left(\mathrm{CDCl}_{3}\right) \delta: 3.43(\mathrm{~d}, 4 \mathrm{H}, J=2.8 \mathrm{~Hz}), 5.58\left(\mathrm{dd}, 1 \mathrm{H}, J_{1}=1.6 \mathrm{~Hz}, J_{2}=1.6 \mathrm{~Hz}\right), 7.19(\mathrm{~s}, 1 \mathrm{H}), 7.32-7.53$ (m, 5H), $7.73(\mathrm{~d}, 1 \mathrm{H}, J=7.2 \mathrm{~Hz}), 8.11(\mathrm{~d}, 1 \mathrm{H}, J=8.4 \mathrm{~Hz}), 9.98(\mathrm{~s}, 1 \mathrm{H})$; LC-MS: $347.6(\mathrm{M}+\mathrm{H})^{+}$; Anal. Calcd. for $\mathrm{C}_{20} \mathrm{H}_{14} \mathrm{~N}_{2} \mathrm{O}_{2} \mathrm{~S}$ (346.40): C, 69.35; H, 4.07; N,8.09\%. Found: C, 69.32; H, 4.05; N, 8.11\%.

\subsection{Cell Culture}

Cell lines MDA-MB-468, SKRB-3 and A375 were maintained in Dulbecco's modified Eagle medium (DMEM) containing 10\% fetal bovine serum (FBS), penicillin $(100 \mathrm{U} / \mathrm{mL})$ and streptomycin $(10 \mathrm{mg} / \mathrm{L})$. Cell lines H460, SW480, and BxPC-3 were maintained in RPMI 1640 containing 10\% FBS, penicillin $(100 \mathrm{U} / \mathrm{mL})$ and streptomycin $(10 \mathrm{mg} / \mathrm{L})$. Cells were grown in a $5 \% \mathrm{CO}_{2}$ incubator at $37^{\circ} \mathrm{C}$.

\subsection{Cell Proliferation Assay (MTT Assay)}

Cells $\left(3-5 \times 10^{3} /\right.$ well) were seeded in $200 \mu \mathrm{L}$ of medium/well in 96-well plates (Costar Corning, Rochester, NY). After incubation overnight, the compounds dissolved in dimethylsulfoxide (DMSO) were added to final concentration of $20 \mu \mathrm{M}$, adriamycin (ADM) used as a positive control. Cells were exposed to the compounds for $48 \mathrm{~h}$. After incubation with 3-(4,5-dimethylthiazol-2-yl)-2,5diphenyltetrazolium bromide (MTT; $0.5 \mathrm{mg} / \mathrm{mL}$ ) for $4 \mathrm{~h}$, the medium was removed and $150 \mu \mathrm{L}$ of 
DMSO was added to dissolve formazan crystals. Absorbance was measured at $570 \mathrm{~nm}$ using an ELISA reader (Thermo). The effects of the compounds on the proliferation of cancer cells were expressed as the $\%$ cell growth inhibition, using the following formula: $\%$ inhibition $=\left(\mathrm{A}_{570}\right.$ of control $-\mathrm{A}_{570}$ of treated cells) $/ \mathrm{A}_{570}$ of control cells $\times 100 \%$.

\section{Conclusions}

In this work, twelve novel acenaphthene derivatives containing thiazole backbone were synthesized and the in vitro antitumor activity was evaluated. Among all of these derivatives, compound 3c showed more potent cytotoxicity towards SKRB-3 cell than the positive control adriamycin, which indicates the potential of $\mathbf{3 c}$ as a future anticancer agent.

\section{Acknowledgements}

This work was supported by China Postdoctoral Science Foundation (20100471652).

\section{References}

1. El-Ayaan, U.; Abdel-Aziz, A.A.-M.; Al-Shihry, S. Solvatochromism, DNA binding, antitumor activity and molecular modeling study of mixed-ligand copper(II) complexes containing the bulky ligand: bis[N-(p-tolyl)imino]acenaphthene. Eur. J. Med. Chem. 2007, 42, 1325-1333.

2. Zhu, W.; Dai, M.; Xu, Y.; Qian, X. Novel nitroheterocyclic hypoxic markers for solid tumor: synthesis and biological evaluation. Bioorg. Med. Chem. 2008, 16, 3255-3260.

3. McDavids, J.E.; Daniels, T.C. The fungistatic properties of acenaphthene derivatives. J. Am. Pharm. Assoc. 1951, 40, 325-326.

4 El-Ayaan, U.; Abdel-Aziz, A.A.-M. Synthesis, antimicrobial activity and molecular modeling of cobalt and nickel complexes containing the bulky ligand: bis[N-(2,6-diisopropylphenyl)imino] acenaphthene. Eur. J. Med. Chem. 2005, 40, 1214-1221.

5. Williamson, W.R.N. Pharmacologically active acenaphthene derivatives. GB2005673A. 1979.

6. Levine, S.D.; Brunswick, N; Harper, I.T.; Park, K. Acenaphthene carboxamides. US Pat. 3732299A, 1973.

7. Eagleson, M. Concise Encyclopedia Chemistry; Walter de Gruyter: Berlin, Germany, 1993; p 3.

8. Gifford, L.A.; Owusu-Daaku, F.T.K.; Stevens, A.J. Acenaphthene fluorescence derivatisation reagents for use in high-performance liquid chromatography. J. Chromatogr. A. 1995, 715, 201-212.

9. Gupta, A.; Mishra, P.; Kashaw, SK.; Jatav, V.; Stables, J.P. Synthesis and anticonvulsant activity of some novel 3-aryl amino/amino-4-aryl-5-imino- $\Delta^{2}-1,2,4$-thiadiazoline. Eur. J. Med. Chem. 2008, 43, 749-754.

Sample Availability: Samples are available from the authors. 
(C) 2011 by the authors; licensee MDPI, Basel, Switzerland. This article is an open access article distributed under the terms and conditions of the Creative Commons Attribution license (http://creativecommons.org/licenses/by/3.0/). 